\title{
Gestión de la cadena de suministro de un programa del Estado: metodologías que se adaptan para su optimización ${ }^{1}$
}

\section{Management of the supply chain of a State program: methodologies that are adapted for its optimization}

\author{
Veralina Tremolada Tovar \\ Pontificia Universidad Católica del Perú \\ veralina.tt@gmail.com
}

\begin{abstract}
El artículo apunta a describir cómo metodologías generalmente utilizadas en el sector privado pueden ser aplicadas para optimizar programas del Estado, y más específicamente, programas de alimentación escolar (PAE). Asimismo, para que su intervención cumpla con sus objetivos $y$, sobre todo, genere valor tanto para los usuarios de los programas como para la sociedad peruana, es imprescindible que la gestión de los PAE se desarrolle de manera eficiente. Es con el fin de aportar a ese cometido que el presente artículo analiza las metodologías Supply Chain Operations Reference (SCOR) y análisis de los interesados del Project Management Body of Knowledge (PMBOK) desde un enfoque teórico aplicativo, las mismas que pueden ser adaptadas o aplicadas directamente según las especificidades de la organización.
\end{abstract}

Palabras clave: metodologías; aplicación; adaptación; especificidades; gestión eficiente

The article aims to describe how methodologies generally used in the private sector can be applied to optimize state programs, and more specifically, school feeding programs (PAE, according to its initials in Spanish). Therefore, to achieve its objectives and, above all, to generate value, both for the users of the programs and for Peruvian society, it is essential that the management of the PAE is developed efficiently. To contribute to this task, the Supply Chain Operations Reference (SCOR) and Stakeholder Analysis of the Project Management Body of Knowledge (PMBOK) are presented from a theoretical application approach, and both methodologies can be adapted or applied directly according to the specificities of the organization.

Keywords: methodologies; application; adaptation; specificities; efficient management

\footnotetext{
1. El artículo presenta las metodologías aplicadas en el proyecto profesional Programa de Alimentación Escolar en Ventanilla: una gestión eficiente bajo la metodología SCOR, el cual fue sustentado por Llubitza Veralina Tremolada Tovar y Vanessa Alexandra Valencia Gómez en diciembre del 2014 para obtener el grado de licenciadas en Gestión. Agradezco a mi compañera Vanessa, con quien recorrí este camino de aprendizaje; y al profesor Germán Velásquez, quien nos guio y retó para lograrlo.
} 


\section{Introducción}

Más de cuatrocientos millones de niños en el mundo viven en pobreza extrema (Banco Mundial, 2013). A raíz de eso, la alimentación que reciben desde sus primeros años de crecimiento no es la más adecuada para su óptimo desarrollo físico y mental, impactando de manera negativa en su nivel de energía, capacidad de aprendizaje, concentración, resultados escolares, voluntad de socialización y asistencia escolar (Cuevas, 2005). Ello, lamentablemente, los mantiene en la pobreza en la mayoría de casos.

La mala nutrición representa un estado poco saludable en el que las funciones del cuerpo de una persona se dañan temporal o permanentemente, perjudicando su crecimiento y adecuado desarrollo. La pobreza es subyacente a la malnutrición en el mundo y ambas se afectan entre sí para formar un círculo vicioso: la pobreza disminuye la capacidad de las personas para conseguir la comida que necesitan o cultivar sus propios alimentos, lo que desemboca en malnutrición; y esta, a su vez, disminuye la capacidad de aprender y trabajar, lo que conduce a incrementar el nivel de pobreza. Esta pérdida de recursos humanos potenciales también se traduce en mayores costos sociales y económicos para la comunidad y el país, afectando significativamente su desarrollo (Menza \& Probart, 2013).

Es por ello que el Estado, con el objetivo de aumentar la capacidad de aprendizaje de los niños, incentivar su asistencia a la escuela y mantener su permanencia (Bundy et al., 2009), crea los programas de alimentación escolar (PAE), que benefician a más de 80 millones de niños en 15 países de la región, invirtiendo aproximadamente USD 4000 millones al año (PMA, 2013). Para que esta inversión de recursos del Estado genere valor, tanto para los niños usuarios como para la sociedad, es crucial que la gestión del programa se desarrolle de manera eficiente. Por tanto, este artículo busca aportar a ese cometido, presentando metodologías que contribuyan al óptimo desempeño de un PAE. En primer lugar, se definirá el concepto de valor dentro de la gestión pública; luego, se comentarán ciertos aspectos relevantes de un PAE; y, finalmente, se definirán y analizarán metodologías del sector privado aplicadas, según sus especificidades, al sector público.

\section{La gestión dentro del Estado: la gestión pública}

\subsection{Generación de valor público}

El Estado, visto como una gran organización, se crea con el fin de satisfacer las demandas ciudadanas; esto es, de generar valor público. Para ello, define qué queremos como sociedad, estableciendo políticas públicas y la forma como las implementamos a través de la gestión pública.

La creación de valor público, como señala Mark Moore (1995), consiste en generar el máximo valor posible para la población a partir de los recursos financieros, humanos, físicos y tecnológicos existentes en la institución, tomando como base la imaginación gerencial, a fin de cumplir con los propósitos establecidos en los mandatos con la máxima eficacia y eficiencia posible (Moore, citado en García, 2015). 
Así pues, las instituciones públicas ejecutan con sus inputs —es decir, con sus recursos- procesos que permiten generar outputs —esto es, actividades plasmadas como bienes, servicios, etcétera-, convirtiéndose estos en el primer objetivo productivo de las instituciones públicas. Dichos outputs producidos, una vez dentro del ámbito de la sociedad, impactan a los beneficiarios, generando outcomes que constituyen el objetivo social de toda la cadena de valor, como se muestra en el siguiente gráfico (García, 2015).

\section{Gráfico 1. La creación de valor público}

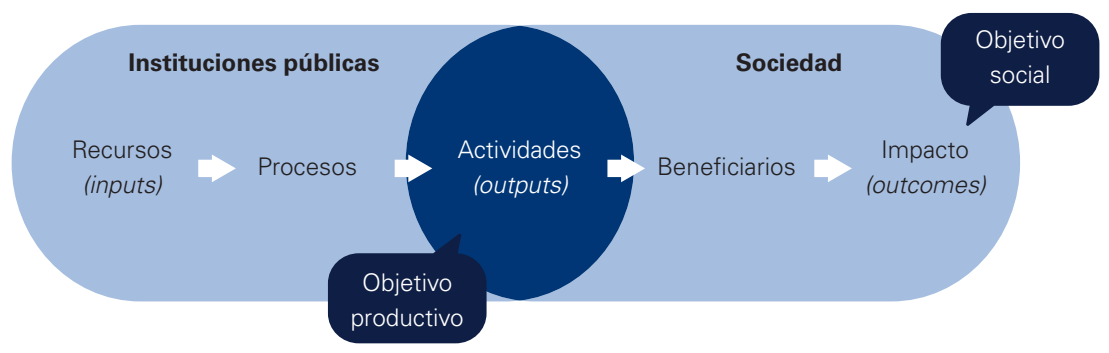

Fuente: García (2015).

Como señala Jorge García en su informe de Gestión pública y valor público:

Hay que tener claro que la oferta de bienes o servicios públicos es solo eso, hasta cuando no haya una apreciación de ellos por parte del público receptor. La oferta de cualquier bien o servicio no necesariamente genera valor; pero sí costos. El valor se genera en la medida que un público reconozca el bien o el servicio como respuesta a una necesidad o preferencia, y aprecie la calidad del bien o servicio como respuesta adecuada a esta $(2015$, p. 6).

\subsection{Programas del Estado: PAE}

De acuerdo a la realidad de cada país, en este caso del Perú, existen problemas o situaciones críticas que el Estado debe afrontar. Para ello, implementa políticas públicas específicas por medio de programas. Es así que nacen, por ejemplo, los PAE, que actúan sobre los diversos factores que influyen en la desnutrición, un obstáculo que impide que los individuos —especialmente en la infancia- e incluso las sociedades desarrollen todo su potencial (UNICEF, s.f.).

La causa principal de la desnutrición es la pobreza (UNICEF, s.f.). En el 2016, el 20,7\% de la población del Perú (6 518000 personas) se encontraba en condición de pobreza (INEI, 2017). Esta condición puede potenciar las otras causas de la desnutrición, como las define UNICEF:

La no lactancia materna exclusiva, la presencia de enfermedades (diarrea, infecciones respiratorias agudas entre otras), la falta de educación y de información sobre la buena o adecuada alimentación, el no consumo de suplementos vitamínicos o alimentos fortificados, y el costo de los alimentos (s.f.). 
La desnutrición o malnutrición que sufren, en este caso, los niños peruanos, provoca o coadyuva a producir efectos como las dificultades de concentración, la carencia de energía, el decaimiento, la disminución de la capacidad de aprendizaje, la escasa voluntad de socialización, los resultados escolares negativos, la deserción escolar y, en general, el retraso del desarrollo (Cuevas, 2005). Por tanto, para evitar la generación de esos efectos, es muy importante que los PAE a implementar cumplan ciertos aspectos determinantes para su óptimo desempeño (Tremolada \& Valencia, 2014):

- El aseguramiento de la calidad e inocuidad de los alimentos a entregar.

- La definición del producto.

- La logística.

- La supervisión.

- La evaluación.

Lamentablemente, los PAE en el Perú se han caracterizado por presentar deficiencias en su gestión; por ejemplo, el primer Programa Nacional de Asistencia Alimentaria (PRONAA), que se extinguió por incoherencias legales, técnicas y de gestión. De la misma manera, durante el primer año de operación del Programa Actual: Programa Nacional de Alimentación Escolar Qali Warma, se han presentado una serie de denuncias por intoxicación de alumnos debido al consumo de alimentos en mal estado, además de una serie de críticas al modelo de gestión (Tremolada \& Valencia, 2014).

Por ello, como ya se mencionó, este artículo busca sugerir herramientas que puedan ser aplicadas para lograr una gestión eficiente en los programas del Estado, pudiendo estas adaptarse a la particularidad de los mismos.

\section{Metodologías del sector privado que se aplican al público}

Una metodología aplicada a cualquier organización —ya sea, por ejemplo, un determinado proyecto empresarial, un caso de estudio social o un programa del Estado- es una estructura de pasos a seguir para lograr un objetivo específico, generalmente de mejora, reestructuración u optimización. En general, dentro de la gestión, las metodologías tienen un enfoque primordialmente empresarial. Y cuando, como gestores, decidimos utilizar una de esas metodologías dentro del sector público, se pueden presentar ciertos cuestionamientos según la esencia de la organización, de modo que se opta por cambiar el modo de aplicación de la metodología. En la siguiente tabla se presentan de forma genérica las características inherentes a las organizaciones privadas y públicas.

Tabla 1. Especificidades de los sectores privado y público

\begin{tabular}{l|l|l} 
& sector privado & sector público \\
\hline Racionalidad & $\begin{array}{l}\text { Orientada a la maximización de beneficios } \\
\text { económicos }\end{array}$ & Depende de los objetivos del Estado
\end{tabular}




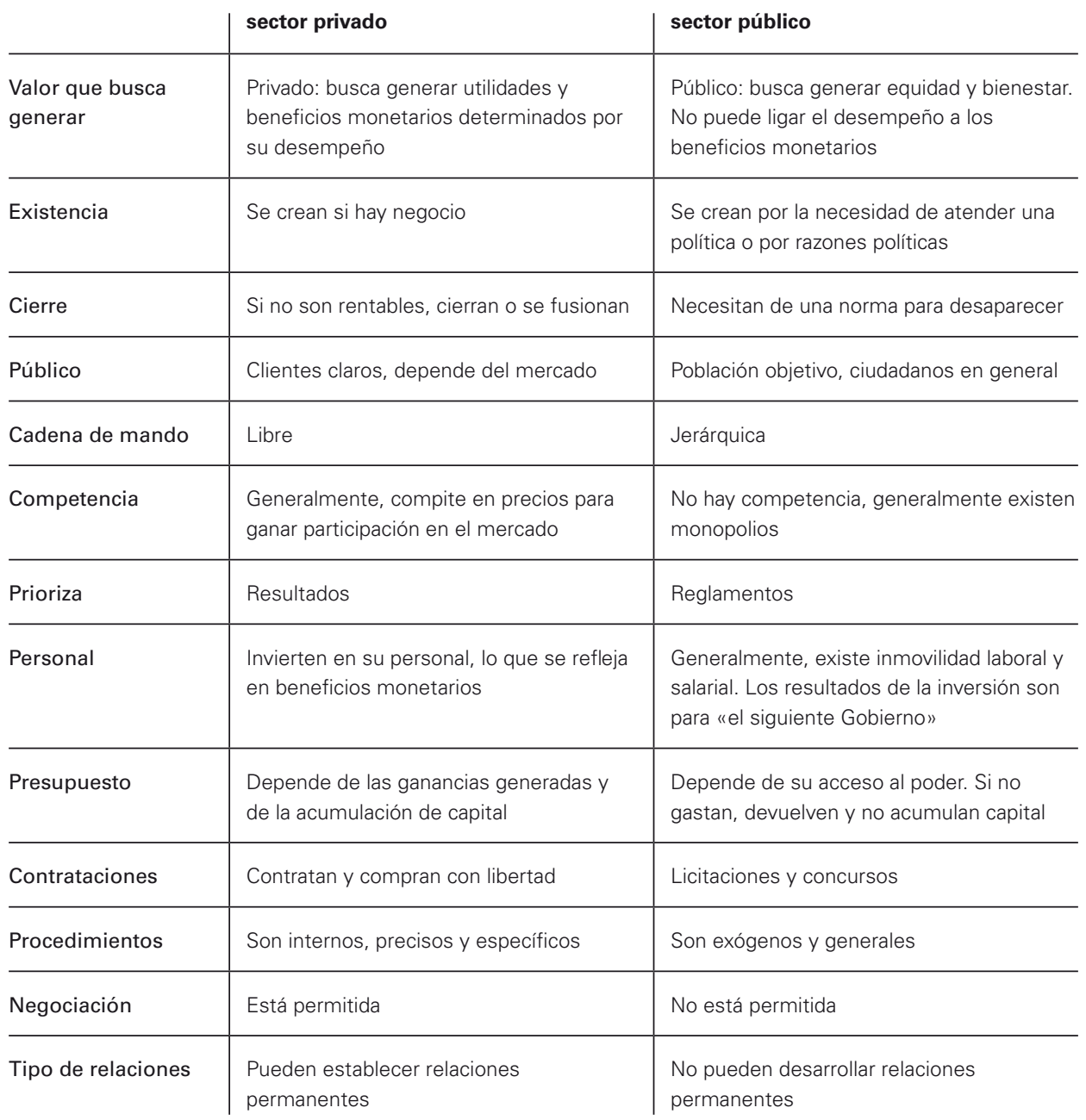

Fuente: adaptado de Ugarte (2011).

Como se observa en la tabla, y según señalan Echebarría y Mendoza (1999), no puede negarse la existencia de condicionantes específicos en el sector público, y si bien estos no lo convierten en un ámbito totalmente diferenciado, sí matizan de forma considerable cualquier desarrollo de las técnicas de gestión.

A continuación, se destacan cuatro aspectos que, por su carácter estructural, confieren una marcada particularidad al contexto en el que tienen lugar los procesos de gestión pública (Echebarría \& Mendoza, 1999):

- La sustitución del mercado por el proceso político como mecanismo de asignación de recursos.

- El hecho de que las administraciones públicas tienen el carácter de poderes públicos.

- La naturaleza distinta de los procesos de creación de valor por parte del sector público.

- La dificultad para medir el valor creado. 
Teniendo en cuenta estas "especificidades que sustentan la diferencia» (Ugarte, 2011) con el sector privado, es indispensable prestar atención a los resultados que las acciones del Estado —en este caso mediante un PAE - causan entre los usuarios, pero también son clave los procesos aplicados para generar dichos resultados, ya que la interacción que se establece entre las organizaciones responsables y la población genera valor por sí misma (García, 2015). Es por ello, que se considera de vital importancia que la implementación de estos programas sea eficiente, lo cual, desde el ámbito de la gestión, nos permite el uso de metodologías que apoyen al sector público en su cometido de generar valor.

Como se muestra en el siguiente gráfico, las técnicas de gestión empresarial se valoran en función de su idoneidad para dar respuesta a los problemas de gestión de las organizaciones públicas. El grado de adecuación de cada grupo de técnicas se expresa en términos de aplicación directa, adaptación creativa o de necesidad de reconceptualización (Echebarría \& Mendoza, 1999).

Gráfico 2. Grado de adecuación de las técnicas de gestión empresarial a la administración pública

\begin{tabular}{l|l|l|l} 
técnica de gestión considerada & aplicación directa & adaptación creativa & reconceptualización \\
\hline Planificación estratégica & & & \\
\hline Gestión del cambio organizativo & & & \\
\hline Dirección por objetivos & & & \\
\hline Dirección de proyectos & & & \\
\hline Gestión de servicios & & & \\
\hline Marketing & & & \\
\hline Dirección de operaciones & & & \\
\hline Diseño organizativo & & & \\
\hline Dirección de recursos humanos & & & \\
\hline Gestión financiera & & & \\
\hline Sistemas de información & & & \\
\hline Control de gestión & & & \\
\hline
\end{tabular}

El gráfico muestra, siguiendo a Echebarría y Mendoza (1999), cómo existe un menor grado de adecuación de las técnicas de gestión empresarial cuando se aplican en el contexto de mayor especificidad de la gestión pública. Esto tiene que ver con la dificultad de conceptualizar y de medir el valor creado, pues afecta tanto a la fijación de objetivos como al control de resultados, lo que, unido a la débil integración del ciclo de gestión (planificaciónejecución-control) en numerosas organizaciones públicas, modifica sustancialmente el carácter de la dirección por objetivos y del control de gestión. Asimismo, la especificidad del estatuto funcionarial otorga una dimensión distintiva a la dirección de recursos humanos y a los procesos de gestión del cambio organizativo (Echebarría \& Mendoza, 1999). Por tanto, 
es clave tener en cuenta la especificidad de las organizaciones públicas para poder aplicar metodologías del sector privado.

Antes de presentar las metodologías seleccionadas, es preciso mencionar la metáfora con la que Eugene Bardach describe la implementación, comparándola con el proceso de ensamblar y mantener en su lugar todos los elementos necesarios de una máquina:

Claro que tal como una máquina puede no funcionar si su diseño es fallido, una política basada en una teoría incorrecta puede producir también consecuencias no intencionadas. Pero un diseño efectivo (teoría correcta) es una condición necesaria pero no suficiente para el desempeño de una máquina (política efectiva). Si las partes necesarias (elementos esenciales de política) no están disponibles o no son confiables, la máquina (política) no trabajará de manera efectiva (citado en Weimer \& Vining, 2011).

Dentro de un PAE, como ya se mencionó, los aspectos determinantes para su desempeño son el aseguramiento de la calidad e inocuidad de los alimentos a entregar, la definición del producto, la logística, la supervisión y la evaluación (Tremolada \& Valencia, 2014). En relación a ello, el informe del Estado de la alimentación escolar a nivel mundial producido por el Programa Mundial de Alimentos (PMA), el Banco Mundial y la Alianza para el Desarrollo Infantil sugiere "elaborar una guía para los países sobre cómo estimar los costos en cada etapa de la cadena de suministro y cómo optimizar las operaciones» (PMA, 2013, p. xv). Así, un diseño y gestión óptima de la "máquina», o de la cadena de suministro del PAE, en este caso, permitirá incrementar la eficiencia y sostenibilidad del mismo (Tremolada \& Valencia, 2014).

Se considera a la cadena de suministro como un elemento integrador dentro de los PAE, ya que busca coordinar eficientemente sus procesos clave para colocar los requerimientos de insumos o productos en cada uno de sus eslabones, como afirman Jiménez y Hernández (2002), en el tiempo preciso y al menor costo, buscando lograr el mayor impacto posible en la cadena de valor de los integrantes con el propósito de satisfacer a los consumidores finales.

La esencia del problema de la implementación recae en la distribución de los elementos necesarios. Mientras mayor sea el potencial de las personas u organizaciones de limitar las contribuciones o el apoyo, mayor es la posibilidad de fallar. Aquellos que se oponen a las metas de la política o aquellos que no ven las metas como suficientemente beneficiosas para justificar sus propios costos de conformidad o aceptación, pueden limitar sus contribuciones o apoyo adrede. Otros pueden hacerlo simplemente debido a que sus recursos y competencias son muy limitados para permitirles intervenir (Weimer \& Vining, 2011).

En relación a ello, cabe resaltar que la cadena de suministro no solo involucra la coordinación del flujo de bienes y servicios, sino también un mayor compromiso de las partes, generando una estrecha vinculación entre los diversos actores de la cadena. Dado que en el desarrollo de la cadena de suministro participan diversos actores, la gestión de relaciones múltiples plantea como uno de sus elementos la estructura de la red de dicha 
cadena, la cual identifica a los miembros dentro de la misma con el objetivo de evaluar qué tan críticos son estos para el éxito de la organización, clasificándolos por nivel (Jiménez \& Hernández, 2002).

"Por lo tanto, dentro de la gestión de la cadena de suministro se deberían analizar los actores estratégicos de la misma, pues son una variable clave para el éxito de la organización» (Tremolada \& Valencia, 2014). Es por ello que se han elegido dos metodologías que permiten analizar la cadena de suministro de un PAE: la Supply Chain Operations Reference (SCOR) y el análisis de los interesados del Project Management Body of Knowledge (PMBOK), las cuales serán detalladas en lo que sigue de este artículo.

\subsection{Supply Chain Operations Reference Model (SCOR)}

SCOR es el modelo de referencia de operaciones de la cadena de suministro desarrollado por el Supply-Chain Council (SCC), y es reconocido a nivel mundial como una herramienta que representa, analiza y configura las cadenas de suministro en una estructura unificada para mejorar su eficacia de gestión (Calderón \& Lario, 2005). Este modelo de proceso de referencia contiene descripciones estándar de los procesos de gestión, un marco de las relaciones entre los procesos estándar, métricas estándar para medir el rendimiento de los procesos, las prácticas de gestión que producen un mejor rendimiento en su clase, y la alineación estándar a las características y la funcionalidad (SCC, 2005).

\subsubsection{Conceptos de la metodología SCOR}

Como se observa en el siguiente gráfico, este modelo de referencia integra los conceptos de benchmarking, reingeniería de procesos empresariales y medición de mejores prácticas en un marco multifuncional (SCC, 2005).

\section{Gráfico 3. Conceptos que integra la metodología SCOR}

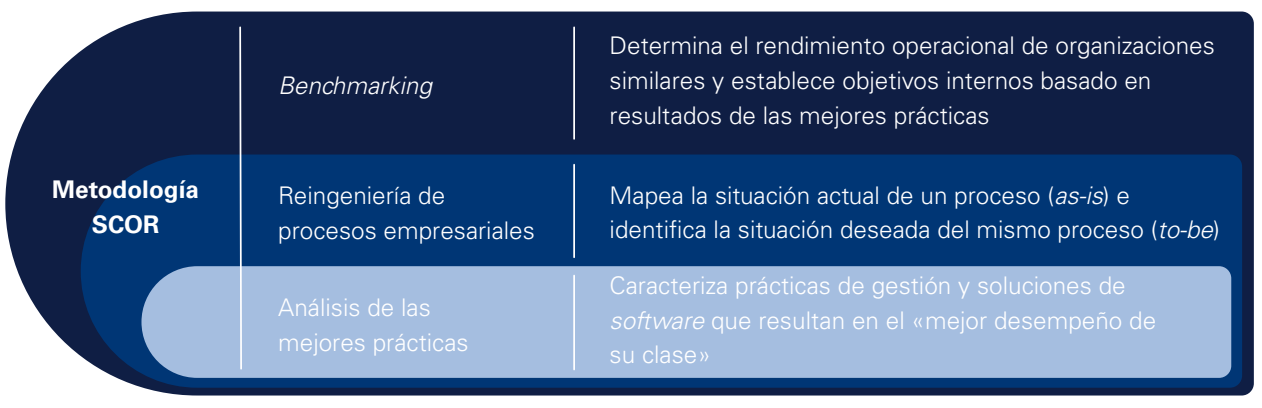

Fuente: adaptado de SCC (2005).

Para cumplir con el desarrollo de la metodología SCOR, estos conceptos se utilizan en las siguientes líneas, a modo de ejemplo, en el análisis de un PAE.

En primer lugar, se aplica el benchmarking para determinar el rendimiento operacional de PAE similares, estableciendo objetivos internos basados en resultados de las mejores prácticas latinoamericanas. Se eligieron los PAE de Latinoamérica no solo porque 
se desarrollan en un contexto similar al de Perú, sino también debido a que, según el informe El estado de la alimentación escolar a nivel mundial 2013, unos 368000000 niños (la quinta parte del total de menores en el mundo) de 169 países desarrollados y en vías de desarrollo reciben comida en sus escuelas todos los días (PMA, 2013). Se trata de una práctica extendida y muy efectiva para combatir la malnutrición, cuya cobertura es mínima en los programas de los países en donde más se necesita, siendo Latinoamérica una excepción.

Entre los ejemplos mundiales, tal como comentó la investigadora Burbano en una entrevista con EFE (Reneé, 24 de mayo de 2013), están Brasil, Chile, Ecuador y México. Estos PAE determinan su rendimiento según la consecución de los siguientes objetivos (Tremolada \& Valencia, 2014):

- Brasil cosecha de la agricultura local para abastecer a sus escuelas.

- Chile externaliza su programa por un servicio de calidad en las escuelas.

- Ecuador cambió a productos que garanticen la efectividad del PAE.

- México promueve una alimentación adecuada para combatir el sobrepeso.

Así, dado que dentro de organizaciones similares se considera a estos PAE como las mejores prácticas, un PAE en el Perú deberá basar sus objetivos internos en los resultados de los PAE elegidos como referentes.

En segundo lugar, hay que mapear la situación de cada proceso de la cadena de suministro del PAE a optimizar (as-is) y, de acuerdo a los problemas y oportunidades de mejora identificados en el análisis de las mejores prácticas, se debe plantear la situación deseada (to-be) de los mismos procesos, considerando prácticas de gestión, sistemas informáticos y soluciones tecnológicas para que el PAE en cuestión desarrolle la gestión de su cadena de suministro de la manera más eficiente posible.

\subsubsection{Procesos de gestión de la metodología SCOR}

La metodología SCOR se basa en cinco procesos básicos de gestión: planeación, abastecimiento, make², distribución y devolución, como se describe a continuación (SCC, 2005):

- Planeación: procesos que equilibren la demanda y oferta agregada para desarrollar un curso de acción que se ajuste mejor a los requisitos de abastecimiento, producción y entrega.

- Abastecimiento: procesos que procuran bienes y servicios para satisfacer la demanda.

- Make: procesos que transforman el producto a su estado final para cumplir con la demanda.

- Distribución: procesos que proporcionan bienes y servicios terminados para satisfacer la demanda, incluyendo típicamente la administración de pedidos, administración de transporte y gestión de la distribución.

2. El proceso make se usa en inglés con el objetivo de evitar traducciones que puedan distorsionar su concepto dentro de la metodología SCOR (Tremolada \& Valencia, 2014). 
- Devolución: procesos asociados con la devolución o recepción de productos devueltos por cualquier razón. Estos procesos se extienden hasta la asistencia al cliente posterior a la entrega.

Estos procesos, como señala la metodología, se dan para todas las transacciones de productos (material físico y servicio), desde el proveedor del proveedor hasta el cliente del cliente, de forma que alcanzan desde la demanda hasta la devolución de productos terminados dentro de la cadena de suministro. Por ello, en el proceso de desarrollo de la metodología SCOR se tienen en cuenta la planificación, gestión de suministros, pedidos, manejo de inventarios, ejecución de la producción, almacenamiento, transporte, gestión de existencias, fabricación del pedido y retorno de materias primas, entre otros, como se detalla en la siguiente tabla (SCC, 2005).

\section{Tabla 2. Alcance de los procesos de la metodología SCOR}

\begin{tabular}{|c|c|}
\hline Planeación & $\begin{array}{l}\text { Demanda/planificación y gestión de suministros } \\
\text { - Equilibrar los recursos con los requisitos y establecer/comunicar los planes para toda la } \\
\text { cadena de suministro } \\
\text { - Gestionar reglas de negocio, rendimiento de la cadena de suministro, recolección de } \\
\text { datos, inventarios, activos fijos, transporte, configuración de la planificación, y requisitos } \\
\text { normativos y de cumplimiento } \\
\text { - Alinear el plan de unidad de la cadena de suministro con el plan financiero }\end{array}$ \\
\hline Abastecimiento & $\begin{array}{l}\text { Fuentes abastecidas, fabricación a pedido y gestión del pedido del producto } \\
\text { - Programar entregas; recibir, verificar y transferir el producto; y autorizar los pagos a } \\
\text { - } \text { - Identificar y seleccionar las fuentes de suministro cuando no estén predeterminadas, } \\
\text { como para la gestión del pedido del producto } \\
\text { - Administrar las reglas de negocio, evaluar el desempeño del proveedor y mantener la data } \\
\text { - Administrar inventario, bienes de capital, productos recibidos, red de proveedores, } \\
\text { importación/exportación, requisitos y acuerdos con proveedores }\end{array}$ \\
\hline Make & $\begin{array}{l}\text { Hacer inventario, fabricar a pedido y ejecutar la producción del pedido } \\
\text { - } \quad \text { Programar actividades de producción, emitir el producto, producir y probar, empaquetar, } \\
\text { organizar producto y liberarlo para la entrega } \\
\text { - Finalizar la gestión del pedido de producto } \\
\text { - Manejo de reglas, rendimiento, datos, productos en proceso (work in progress [WIP]), } \\
\text { equipos e instalaciones, transporte, red de producción y cumplimiento de normativas } \\
\text { para la producción }\end{array}$ \\
\hline Distribución & $\begin{array}{l}\text { Orden, almacenamiento, transporte e instalación de gestión de las existencias, } \\
\text { fabricación a pedido y gestión del pedido del producto } \\
\text { - Todas las etapas de gestión de pedidos, desde el procesamiento de consultas y } \\
\text { cotizaciones de clientes hasta el enrutamiento de envíos y la selección de transportistas } \\
\text { - Gestionar almacenes desde la recepción y selección del producto hasta la carga y envío } \\
\text { - del mismo } \\
\text { - Recibir y verificar el producto en el sitio de cliente e instalar, si es necesario } \\
\text { - Gestionar la entrega de reglas de negocio, rendimiento, información, inventarios de } \\
\text { productos terminados, bienes de capital, transporte, ciclo de vida del producto y } \\
\text { requisitos de importación/exportación }\end{array}$ \\
\hline
\end{tabular}


Retorno de materias primas y recepción de devoluciones de productos terminados

- Todas las etapas de devolución de productos por defectos; por mantenimiento, reparación y revisión; y por excedente, desde el abastecimiento —identificación de la condición del producto, disposición del producto, solicitud de autorización de devolución del producto, envío del producto programado, y devolución del producto defectuoso/

Devolución producto $\mathrm{MRO}$ */exceso del producto— hasta la entrega — la devolución autorizada del producto, el recibo de entrega, el producto recibido y la transferencia del producto defectuoso/producto MRO/exceso del producto-

- Administrar reglas empresariales de devolución, rendimiento, recopilación de datos, inventario de devolución, activos de capital, transporte, configuración de la red, y requisitos y cumplimiento normativos

* Se refiere al mantenimiento, reparación y operación. Fuente: adaptado de SCC (2005).

Como se puede deducir, la tabla anterior se centra en los procesos que desarrolla una empresa; sin embargo, conocer al detalle el alcance de cada uno de ellos contribuye a adaptar el modelo a cualquier tipo de organización y, en este caso, al análisis de la cadena de suministro de un programa del Estado. Para ejemplificar ello, se muestra la adaptación de los cinco procesos en los que se basa la metodología SCOR para describir y analizar específicamente la cadena de suministro de un PAE, según el proyecto profesional Programa de Alimentación Escolar en Ventanilla: una gestión eficiente bajo la metodología SCOR, definidos como sigue (Tremolada \& Valencia, 2014):

- Planeación: describe la planificación del programa en cuanto a los recursos disponibles y a la población por atender, lo cual influirá en el desarrollo de las actividades de los próximos procesos.

- Abastecimiento: describe el proceso de adquisición de los desayunos escolares del programa, desde la definición del proceso de compra hasta el monitoreo de la elaboración de los productos.

- Make: describe las acciones complementarias que el programa realiza para cumplir con sus objetivos estratégicos, tales como las capacitaciones, evaluaciones, comunicaciones, etcétera.

- Distribución: describe la entrega de los desayunos escolares a las instituciones educativas y usuarios del programa.

- Devolución: describe los procedimientos desarrollados ante casos de devolución, quejas y reclamos sobre el servicio de alimentación escolar del programa.

\subsubsection{Niveles de detalle de procesos de la metodología SCOR}

La metodología SCOR representa, analiza y configura la cadena de suministro según tres niveles de detalle. El primer nivel considera los tipos de procesos; el segundo determina las categorías de procesos; y el tercero descompone los procesos. Dentro del modelo se menciona que existe un cuarto nivel3; sin embargo, el SCC (2005) reconoce que no

3. El cuarto nivel es el de implementación (elementos de proceso de descomposición), donde las empresas aplican prácticas de gestión de la cadena de suministro específicas a este nivel. El cuarto nivel define las prácticas para lograr una ventaja competitiva y adaptarse a las cambiantes condiciones del negocio (SCC, 2005) 
está al alcance de la metodología, razón por lo cual se consideran solo los primeros tres niveles mencionados.

\section{Gráfico 4. Niveles de detalle de los procesos según la metodología SCOR}

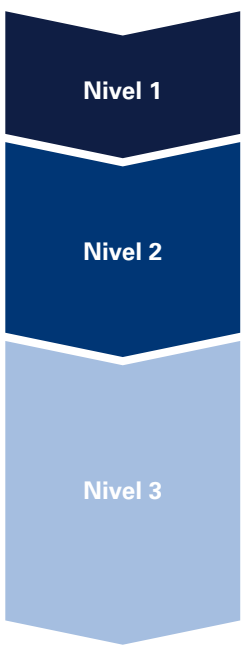

Nivel superior (tipos de procesos): define el alcance y contenido de la aplicación de SCOR. En este nivel es donde se definen los objetivos de rendimiento competitivo

Nivel de configuración (categorías de proceso): la cadena de suministro de una empresa puede ser "configurada a pedido" en el segundo nivel de las "categorías de proceso" principales. Las empresas implementan su estrategia de operaciones a través de la configuración que eligen para su cadena de suministro

Nivel de elementos del proceso (descomposición de procesos): define la capacidad de una empresa para competir exitosamente en los mercados elegidos y consiste en: definición de elementos de procesos; información de elementos de procesos de entrada y salida; métricas de rendimiento de procesos; las mejores prácticas, cuando sean aplicables; las capacidades del sistema necesarias para soportar las mejores prácticas; y los sistemas/herramientas. Las compañías "ajustan» su estrategia de operaciones en este tercer nivel

Fuente: adaptado de SCC (2005).

Según la aplicación de la metodología se deben disgregar los procesos del PAE, lo cual ayudará a identificar los logros y problemas del programa. Esto es imprescindible para definir los elementos de los procesos a reconfigurar. El análisis consiste en identificar los objetivos de los procesos y la jerarquía de los mismos para después definir las políticas y estrategias necesarias para cumplir con los objetivos de cada proceso (SCC, 2005), tal como se ve en el siguiente gráfico:

\section{Gráfico 5. Lógica de descomposición de los procesos según la metodología SCOR}

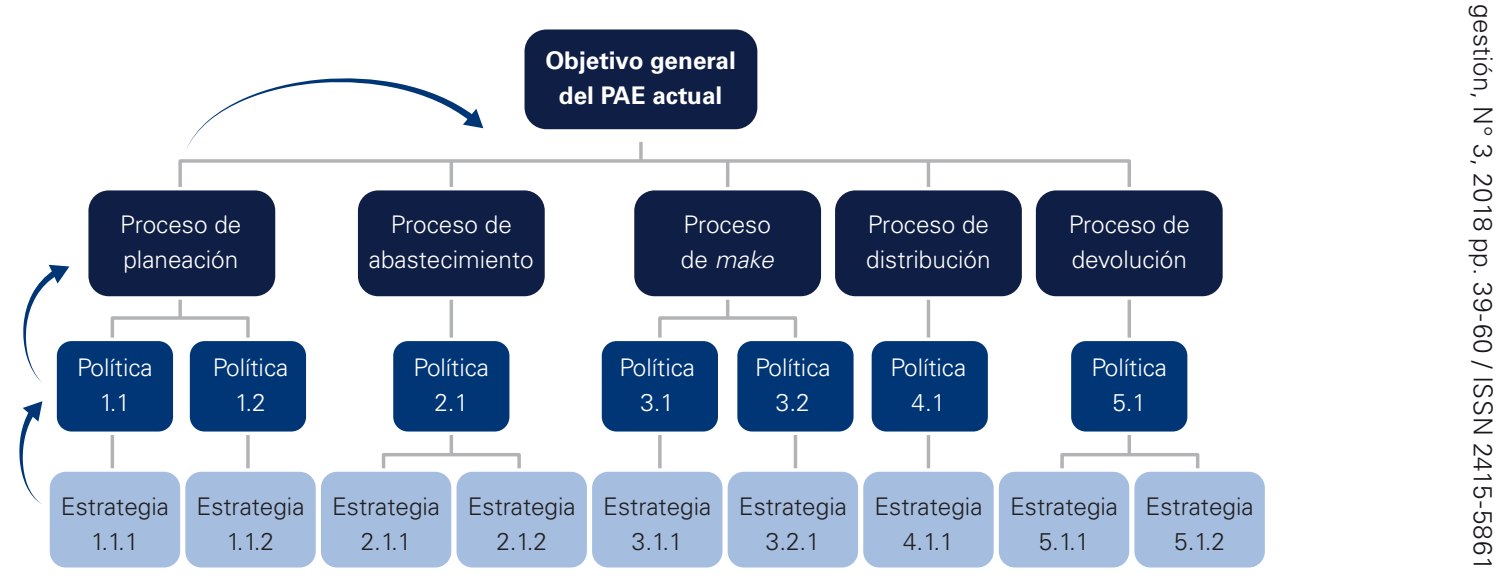

Gráficamente, se puede observar de abajo hacia arriba que el cumplimiento de las estrategias planteadas llevará al adecuado desarrollo de las políticas definidas por el 
programa, con lo cual se deberían alcanzar los objetivos de cada proceso de la cadena de suministro, cumpliendo por ende con el objetivo general del PAE. Es en esta última fase donde se puede apreciar la importancia del adecuado desenvolvimiento de cada parte de la estructura propuesta por la metodología, que al final lleva a cumplir el objetivo de manera integral.

Siguiendo el análisis SCOR, como ya se explicó conceptualmente, identificamos los logros, problemas y oportunidades de mejora según los niveles de detalle, además de definir los elementos de los procesos del PAE a reconfigurar. Así, después de identificar los objetivos de los procesos de gestión dentro de la cadena de suministro — planeación, abastecimiento, make, distribución y devolución-y de definir las políticas y estrategias necesarias para cumplir con los objetivos de cada proceso (SCC, 2005), se procede a configurar las categorías de proceso. Aquí es donde, según el estado actual (as is) de la cadena de suministro del PAE en análisis, se establece el estado deseado (to be) de la misma por medio de la lógica de descomposición de procesos.

Seguidamente, para comprender mejor esta lógica de descomposición, se presenta, a modo de resumen, la definición del estado to be a partir del análisis as is del proceso make del PAE analizado en el proyecto profesional Programa de Alimentación Escolar en Ventanilla: una gestión eficiente bajo la metodología SCOR (Tremolada \& Valencia, 2014).

Tabla 3. Configuración de categorías del proceso make del PAE

\begin{tabular}{|c|c|c|c|c|}
\hline PAE & \multicolumn{2}{|l|}{ estado as is } & \multicolumn{2}{|l|}{ estado to be } \\
\hline Objetivo & \multicolumn{2}{|c|}{$\begin{array}{l}\text { Gestión de las condiciones claves del } \\
\text { programa en materia de transferencia } \\
\text { de conocimientos, comunicaciones y } \\
\text { seguimiento-evaluación }\end{array}$} & \multicolumn{2}{|c|}{$\begin{array}{l}\text { Gestión de las condiciones claves del } \\
\text { programa en materia de transferencia de } \\
\text { conocimientos, comunicaciones y monitoreo- } \\
\text { seguimiento-evaluación }\end{array}$} \\
\hline \multirow{4}{*}{$\begin{array}{l}\text { Políticas y } \\
\text { estrategias }\end{array}$} & \multirow{2}{*}{$\begin{array}{l}\text { 1. El programa } \\
\text { desarrolla un } \\
\text { componente } \\
\text { educativo para } \\
\text { incrementar la } \\
\text { eficacia de los } \\
\text { actores involucrados }\end{array}$} & $\begin{array}{l}\text { 1.1 Brindar asistencia } \\
\text { técnica a los } \\
\text { principales actores } \\
\text { del PAE }\end{array}$ & \multirow{2}{*}{$\begin{array}{l}\text { 1. El programa } \\
\text { desarrolla un } \\
\text { componente } \\
\text { educativo para } \\
\text { incrementar la } \\
\text { eficiencia de los } \\
\text { actores involucrados }\end{array}$} & $\begin{array}{l}\text { 1.1 Brindar } \\
\text { capacitaciones } \\
\text { y evaluar a los } \\
\text { encargados } \\
\text { del servicio de } \\
\text { alimentación escolar }\end{array}$ \\
\hline & & $\begin{array}{l}1.2 \text { Utilización } \\
\text { de herramientas } \\
\text { educativas }\end{array}$ & & $\begin{array}{l}\text { 1.2 Desarrollo } \\
\text { de herramientas } \\
\text { educativas }\end{array}$ \\
\hline & \multirow[t]{2}{*}{$\begin{array}{l}\text { 2. El programa } \\
\text { realiza un plan de } \\
\text { comunicaciones }\end{array}$} & \multirow[t]{2}{*}{$\begin{array}{l}2.1 \text { Utilización } \\
\text { de herramientas } \\
\text { comunicativas }\end{array}$} & \multirow[t]{2}{*}{$\begin{array}{l}\text { 2. El programa } \\
\text { se posiciona } \\
\text { positivamente en la } \\
\text { sociedad y mantiene } \\
\text { una comunicación } \\
\text { constante }\end{array}$} & $\begin{array}{l}2.1 \text { Realización } \\
\text { de un plan de } \\
\text { comunicaciones } \\
\text { con énfasis en el } \\
\text { posicionamiento del } \\
\text { programa }\end{array}$ \\
\hline & & & & $\begin{array}{l}2.2 \text { Desarrollo de un } \\
\text { portal de transparencia } \\
\text { PAE }\end{array}$ \\
\hline
\end{tabular}




\begin{tabular}{|c|c|c|c|c|}
\hline PAE & \multicolumn{2}{|l|}{ estado as is } & \multicolumn{2}{|l|}{ estado to be } \\
\hline \multirow[t]{2}{*}{$\begin{array}{l}\text { Políticas y } \\
\text { estrategias }\end{array}$} & \multirow[t]{2}{*}{$\begin{array}{l}\text { 3. El programa } \\
\text { evaluará su } \\
\text { desempeño }\end{array}$} & \multirow[t]{2}{*}{$\begin{array}{l}\text { 3.1 Desarrollo de } \\
\text { una evaluación de } \\
\text { impacto }\end{array}$} & \multirow{2}{*}{$\begin{array}{l}\text { 3. El programa } \\
\text { implementa } \\
\text { un sistema } \\
\text { de monitoreo, } \\
\text { seguimiento y } \\
\text { evaluación }\end{array}$} & $\begin{array}{l}3.1 \text { Implementación } \\
\text { de un sistema de } \\
\text { monitoreo informático } \\
\text { SIGPAE en las } \\
\text { plantas proveedoras } \\
\text { e instituciones } \\
\text { educativas }\end{array}$ \\
\hline & & & & $\begin{array}{l}\text { 3.2 Desarrollo de } \\
\text { estudios de gabinete } \\
\text { anuales y una } \\
\text { evaluación de impacto }\end{array}$ \\
\hline
\end{tabular}

Fuente: adaptado de Tremolada y Valencia (2014).

Como se puede observar en la tabla anterior, en el estado to be el objetivo del PAE se mantiene similar; sin embargo, las políticas se definen de forma más específica y las estrategias se consideran con mayor detalle con el fin de que los pasos a cumplir para la consecución del objetivo del PAE se implementen de manera eficiente. Ahora, con este nuevo estado de procesos, se puede representar la propuesta según la metodología SCOR para un PAE. La lógica de descomposición descrita permite visualizar el objetivo que debe cumplir cada proceso, así como las políticas, estrategias y definición de variables clave. Estas últimas no se observan en la figura, pero es recomendable considerarlas como condicionantes que, por medio de los indicadores clave de desempeño o KPI (Key Performance Indicators), permitirán medir el cumplimiento de las estrategias definidas para cada política dentro de los cinco procesos mencionados. Estos indicadores se presentan ordenados en una tabla después de cada política y cada uno constará de la siguiente información:

- Nombre de la política.

- Nombre de la estrategia.

- Variable a medir.

- Nombre del indicador.

- Alcance (quiénes intervienen en el desarrollo del indicador).

- Descripción (meta).

- Fórmula.

- Fuente de verificación.

- Plazo (número de veces que el indicador es medido).

- Plan de acción en caso se presente desviación; es decir, no se logre cumplir con la meta establecida.

Para ejemplificar el diseño de los KPI se presenta la siguiente tabla, extraída de la política 3 del proceso make, definida en el proyecto profesional (Tremolada \& Valencia, 2014). 
Tabla 4. KPI 1 de la política 3 del proceso make del PAE

\section{Política 3) El programa implementa un sistema de monitoreo, seguimiento y evaluación}

Estrategia 1: implementación de un sistema de monitoreo informático SIGPAE*

\begin{tabular}{|c|c|}
\hline Variable & Plantas proveedoras e instituciones educativas usuarias controladas en el SIGPAE \\
\hline Nombre & $\begin{array}{l}\text { Porcentaje de plantas proveedoras e instituciones educativas usuarias controladas en } \\
\text { el SIGPAE }\end{array}$ \\
\hline Alcance & $\begin{array}{l}\text { Sede central del PAE propuesto, unidad territorial (UT) del PAE propuesto, los } \\
\text { supervisores y encargados del programa }\end{array}$ \\
\hline Descripción & $\begin{array}{l}\text { El } 100 \% \text { de las plantas proveedoras e instituciones educativas usuarias son } \\
\text { controladas en el SIGPAE }\end{array}$ \\
\hline Fórmula & $\begin{array}{l}\text { ( } N^{\circ} \text { de plantas proveedoras e instituciones educativas usuarias controladas en el } \\
\text { SIGPAE / } N^{\circ} \text { total de instituciones educativas usuarias y plantas proveedoras)* } 100\end{array}$ \\
\hline Fuente de verificación & $\begin{array}{l}\text { Informes y reportes del SIGPAE sobre la actualización de la información de las } \\
\text { instituciones educativas usuarias y plantas proveedoras }\end{array}$ \\
\hline Plazo & Trimestral \\
\hline Plan de acción & $\begin{array}{l}\text { Identificar al actor (supervisor o encargado) que actualiza y automatiza la información } \\
\text { de la institución educativa usuaria —o a la planta proveedora no controlada en el } \\
\text { SIGPAE_- para que sea reemplazado por otro actor que cumpla, en mayor medida, } \\
\text { con el perfil requerido del puesto }\end{array}$ \\
\hline
\end{tabular}

* Sistema Integrado de Gestión del PAE. Fuente:Tremolada y Valencia (2014).

A lo largo de la representación, análisis y configuración de la cadena de suministro, y de acuerdo a los niveles de aplicación de la metodología SCOR, se desarrollan interacciones entre los actores; por lo tanto, como se mencionó al inicio, el análisis de los interesados del PMBOK, en este caso aplicado de forma directa a un PAE, es utilizado como insumo para definir a los actores estratégicos que formarán parte de la cadena de suministro del programa. A continuación, se detalla esta metodología.

\subsection{Análisis de los interesados del PMBOK}

El Project Management Body of Knowledge (PMBOK) es una guía publicada por el Project Management Institute ${ }^{4}$ (PMI) para el conocimiento de la gestión de proyectos. Es internacionalmente reconocida como un recurso fundamental para la gestión efectiva de proyectos en cualquier industria (PMI, 2018).

EI PMBOK no debe entenderse como una metodología por sí misma, sino como una pauta para que los profesionales puedan adaptar a cada caso y contexto particular los

4. El Project Management Institute (PMI) es la principal asociación de membresía profesional sin fines de lucro para la gestión de proyectos (PMI, 2018). 
procesos reconocidos como buenas prácticas por el PMI. Su importancia radica en que provee un marco de referencia formal para desarrollar proyectos, guiando y orientando a los gerentes de proyectos sobre la forma de avanzar en los procesos para la construcción de resultados y la consecución de los objetivos (Colmenares, 2012).

Dentro de la quinta ${ }^{5}$ versión del PMBOK se encuentra el análisis de los interesados. Este análisis es presentado como una técnica que ayuda a recopilar y examinar de manera sistemática información cualitativa y cuantitativa, la cual permitirá determinar los intereses de actores específicos que deben tenerse en cuenta a lo largo del proyecto (PMI, 2013). Para ello, resulta fundamental identificar a los actores interesados desde el comienzo del mismo y analizar sus niveles de interés, expectativa, importancia e influencia. De esa forma, por medio de este análisis, se elabora una estrategia de gestión de actores a fin de maximizar sus influencias positivas y mitigar sus impactos negativos potenciales. Asimismo, es preciso mencionar que dicha estrategia debe revisarse de forma periódica durante la ejecución del proyecto para que, en caso de eventuales cambios, pueda ser ajustada (PMI, 2009).

Este análisis de actores estratégicos, según Melissa Kantor, especialista en gestión de proyectos del PMI, consta de cuatro pasos (2006): identificar, clasificar, entender las necesidades y programas de trabajo, y comprender las relaciones entre los actores.

\subsubsection{Identificar a los actores estratégicos}

El primer paso del análisis es identificar quiénes son los actores estratégicos. Un actor estratégico es cualquier persona afectada directa o indirectamente por el proyecto, o aquel que tiene interés en este (Kantor, 2006). Por ello, para identificar actores estratégicos en un PAE, se debe realizar una descripción detallada tanto de las organizaciones como de las personas que interactúan directa o indirectamente con el programa.

\subsubsection{Clasificar a los actores estratégicos}

Tras identificar a todos los actores estratégicos, el siguiente paso es clasificarlos. Existen múltiples modelos de clasificación utilizados para el análisis de las partes interesadas, tales como (PMI, 2013):

- Matriz de poder/interés, que agrupa a las partes interesadas en función de su nivel de autoridad («poder») y su nivel de preocupación («interés») con respecto a los resultados del proyecto.

- Matriz de poder/influencia, que agrupa a las partes interesadas en función de su nivel de autoridad («poder») y su participación activa («influencia») en el proyecto.

- Matriz de influencia/impacto, que agrupa a las partes interesadas en función de su participación activa ( influencia») y su capacidad para efectuar cambios en la planificación o ejecución del proyecto («impacto»).

5. Durante el proceso de publicación de este artículo, el PMI publicará la sexta edición del PMBOK, la más reciente para el año 2018. 
- Modelo de prominencia, que describe las clases de actores basándose en su poder (capacidad de imponer su voluntad), urgencia (necesidad de atención inmediata) y legitimidad (su participación es apropiada).

Para el análisis de los actores estratégicos dentro de un PAE es recomendable recopilar y analizar de manera sistemática información cualitativa mediante entrevistas a los actores. Para ello, se considera pertinente el uso de la matriz poder/interés del PMBOK 2013, que —como se mencionó- agrupa a los interesados basándose en su nivel de autoridad («poder») y su nivel de preocupación («interés»), siendo clave relacionarlos con la finalidad del proyecto.

Asimismo, para clasificar a los actores estratégicos dentro de la matriz se realiza una ponderación y segmentación de los actores identificados. Es relevante mencionar que, según Kantor (2006), se debe identificar a personas como los actores estratégicos $-\mathrm{y}$ no a las organizaciones o los departamentos- pues una persona específicamente debe ser el punto de contacto para obtener la información. Por eso, sugiere que la información recabada para el uso de la metodología de análisis de los interesados se centre específicamente en personas clave y no en el conjunto de actores u organizaciones dentro de los procesos de la cadena de suministro.

A partir del levantamiento de información cualitativa se pondera el nivel de poder e interés de los actores estratégicos, tomando como referencia los valores del 1 al 4, como se ve en la siguiente tabla (Tremolada \& Valencia, 2014).

Tabla 5. Valores de poder e interés ponderados para los actores estratégicos

\begin{tabular}{l|l} 
valor & significado de poder/interés \\
\hline 1 & Nada \\
\hline 2 & Poco \\
\hline 3 & Moderado \\
\hline 4 & Mucho
\end{tabular}

Fuente: Tremolada y Valencia (2014).

Cabe precisar que el PMBOK recomienda recurrir al juicio de expertos para decidir sobre el nivel de participación requerido de cada uno de los interesados en las diferentes etapas del proyecto (PMI, 2013). Por ello, es importante que la ponderación asignada sea validada por quien mantiene una relación cercana con los demás actores estratégicos del programa.

De acuerdo a la ponderación de poder e interés definida para cada actor estratégico, se construye una matriz de poder/interés de los actores estratégicos del PAE como la que se muestra a continuación en el gráfico 6. 


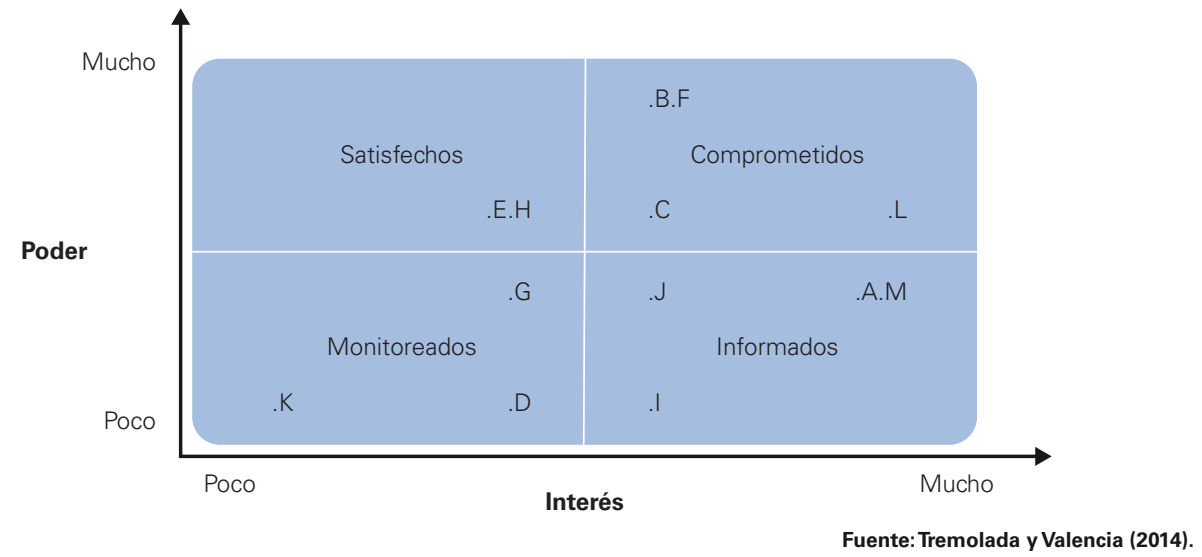

Como se observa en la matriz, existen diversos tipos de actores estratégicos en el programa, los cuales se representan con letras para facilitar el análisis. Dentro de los actores identificados existen cuatro grupos: el grupo que debe mantenerse satisfecho, el que hay que gestionar atentamente para mantenerlo comprometido, el que debe ser monitoreado y el que debe mantenerse informado (PMI, 2013). A continuación, se describe el tratamiento que debe recibir cada uno de los actores según el cuadrante de la matriz en el que se encuentre.

- Mantener comprometidos: los actores estratégicos que están en el cuadrante superior derecho son los que muestran mayor interés y poder dentro del programa. Así, según la especialista Kantor (2006), estos son los actores estratégicos clave; es decir, los actores estratégicos más importantes, y es necesario mantenerlos comprometidos.

- Mantener satisfechos: estos actores estratégicos también tienen una gran influencia dentro del programa; sin embargo, no se muestran muy interesados en el desarrollo del mismo. Por ello, Kantor (2006) los considera como actores difíciles de tratar, ya que, de mantenerse insatisfechos, pueden generar un gran impacto negativo, por lo que se recomienda comprometerlos para comprender sus necesidades y, de esta manera, asegurar su satisfacción.

- Mantener informados: los actores estratégicos que se encuentran en la esquina derecha inferior tienen un gran interés en el programa, pero un poder bastante limitado dentro de este. Por ello, se deben mantener informados oportunamente, pues podrían proporcionar un aporte valioso (Kantor, 2006).

- Mantener monitoreados: por último, dentro del cuadrante inferior izquierdo se ubican los actores que tienen poco interés en el programa y un bajo poder dentro de este. Se recomienda mantener monitoreado e informado a este grupo de actores, evitando las comunicaciones excesivas; y, además, estar atentos a su desenvolvimiento en caso de que sus grados de poder o interés se incrementen (Kantor, 2006). 


\subsubsection{Entender las necesidades y los programas de trabajo de los actores estratégicos}

El tercer paso consiste en comprender a los interesados clave. Por tratarse de actores críticos para el adecuado desarrollo del proyecto, es importante saber cómo se sienten y reaccionan a este. Igualmente, se debe conocer la manera más adecuada de hacerlos participar y de comunicarse con ellos. El mejor método para identificar y entender las necesidades y los programas de trabajo de los actores estratégicos es la toma de entrevistas personales, las cuales permitirán conocer a aquellos actores que podrían apoyar u oponerse al proyecto (Kantor, 2006). Al realizar el trabajo de campo se deben identificar las necesidades de los actores estratégicos y designar a cada uno como "defensor», "neutro" o "bloqueador», según su actitud percibida hacia el logro de los objetivos del programa (Thompson, s.f.). A continuación, se describe cada tipo de actor:

- Actor defensor: es un apoyo crucial en el logro de objetivos del programa.

- Actor neutro: en el momento, no presenta mayor impacto; no obstante, es un actor que debe tenerse en cuenta pues sus necesidades pueden cambiar.

- Actor bloqueador: es crítico en el desarrollo del servicio y, por lo tanto, su permanencia o las reglas de juego con las que se maneja deben ser reconsideradas.

\subsubsection{Comprender las relaciones entre los actores estratégicos}

El cuarto paso del análisis consiste en comprender las relaciones entre los actores estratégicos. Este paso permite entender mejor cómo se relacionan los actores entre sí para, de esa manera, poder manejar sus expectativas. Todo ello con el objetivo de asegurar que los actores positivos mantengan su actitud, reducir los efectos de los negativos, y mejorar la persuasión y comunicación (Kantor, 2006).

Estas relaciones se pueden analizar detalladamente según los procesos de gestión de la metodología SCOR — planeación, abastecimiento, make, distribución y devolucióncon el objetivo de visualizar en cuál de los procesos se genera cada una. De esa forma se podrá identificar qué actores mantienen un mayor número de relaciones; es decir, quiénes participan de manera más activa en alguno de los procesos de gestión. Por ello, resulta relevante considerar el entorno de los actores estratégicos dentro de la cadena de suministro pues permitirá comprenderlos y dirigirlos de la manera más adecuada, teniendo en cuenta sus expectativas, competencias y sinergias (Kantor, 2006).

La adecuada aplicación de la metodología de análisis de los interesados dentro de un programa del Estado resulta fundamental pues, como se señaló, durante el desarrollo de la cadena de suministro participan diversos actores (Jiménez \& Hernández, 2002), de los cuales dependerá la gestión del programa, y la consecución —o no- de sus objetivos.

\section{Conclusiones}

El uso de metodologías generalmente aplicadas al sector privado, como SCOR y el análisis de los interesados del PMBOK, aportan a la generación de valor de las organizaciones del 
Estado. Si su aplicación se realiza de manera adecuada, pueden ayudar a diseñar un PAE eficiente u optimizarlo, según sea el caso.

Además, como se señala a lo largo del artículo, ambas metodologías se pueden aplicar a programas del Estado, en este caso a los PAE. Sin embargo, se deben tener en cuenta sus especificidades antes de aplicarlas directamente, pues esta aplicación puede necesitar ser adaptada según las características de la organización y su propósito a cumplir.

Por otro lado, la metodología SCOR se puede aplicar a programas del Estado, siendo configurada de acuerdo a las necesidades de la organización. En el caso de un PAE, primero se amolda el alcance de los procesos de la metodología según su cadena de suministro para luego disgregar los procesos, siguiendo la lógica de descomposición de la metodología.

En el caso del análisis de los interesados del PMBOK, este tiene una aplicación directa en un programa del Estado, ya que los elementos de la metodología concuerdan con el proceso de identificación y análisis de actores de un PAE.

Por último, es necesario resaltar que dentro de la cadena de suministro de una organización se producen relaciones entre actores. Es por ello que, como se mostró con el PAE, el análisis de la cadena de suministro basado en la metodología SCOR se complementa efectivamente con el mapeo de actores estratégicos de la metodología de análisis de los interesados del PMBOK. 


\section{bibliografía}

Bundy, D.; C. Burbano,

M. Grosh, A. Gelli, M. Jukes

\section{\& L. Drake}

2009

Reconsiderando la alimentación escolar: programas de protección social, desarrollo infantil y el sector educativo. Washington,

D.C.: Banco Mundial.

\section{Calderón, J. \& F. Lario}

2005

Análisis del modelo SCOR para la

gestión de la cadena de suministro. IX

Congreso de Ingeniería de Organización,

1-10. Recuperado de: <http://adingor.

es/congresos/web/uploads/cio/cio2005/

cadena suministros/41.pdf>.

\section{Cuevas, $\mathbf{R}$.}

2005

El diseño de los programas de

alimentación escolar y la función de la

industria alimentaria. En Alimentación,

nutrición y Agricultura. FAO. Recuperado

de: <http://www.fao.org/docrep/008/

y5906m/y5906m05.htm >.

\section{Colmenares, $\mathrm{A}$.}

s.f. ¿Qué es el PMI y qué es el

PMBOK? Recuperado de: <https://

formulaproyectosurbanospmipe.

wordpress.com/2012/01/18/que-es-el-pmiy-que-es-el-pmbok/> (consultado el $18 \mathrm{de}$ enero de 2012).

\section{Echebarría, K. \&}

\section{Mendoza}

1999 La especificidad de la gestión pública: el concepto del management público. En ¿De burócratas a gerentes? Las ciencias de la gestión aplicadas a la administración del Estado (cap.1). Washington, D.C.: Banco Interamericano de Desarrollo.

\section{Fondo de las Naciones Unidas} para la Infancia (UNICEF)

s.f. Desnutrición infantil. Recuperado

de: <http://www.unicef.org/

republicadominicana/health

childhood 10172.htm>.

\section{Jiménez, J. \& S. Hernández}

2002

Marco conceptual de la cadena de

suministro: un nuevo enfoque logístico. Instituto Mexicano del Transporte, (215),

1-272. Recuperado de: <http://imt.mx/ archivos/Publicaciones/PublicacionTecnica/ pt215.pdf >

\section{Instituto Nacional de} Estadística e Informática (INEI)

En el Perú 264 mil personas dejaron de ser pobres entre los años 2015 y 2016. Recuperado de: <https://www.inei.gob. pe/prensa/noticias/en-el-peru-264-milpersonas-dejaron-de-ser-pobres-entre-losanos-2015-y-2016-9710/>.

\section{Kantor, M.}

La clave para un proyecto exitoso de $\mathrm{RRHH}$ : análisis de los actores estratégicos.

En Society for human resource management. Recuperado de: < http:// www.shrm.org/foreign/espanol/Pages/ Article05 hrproject.aspx>.

\section{García, H.}

2015 Gestión pública y valor público. Actualidad Gubernamental, (78).

\section{Moore, M.}

2015 Creating Public Value - Strategic Management in Government. Cambridge: Harvard University Press. 


\section{bibliografía}

Programa Mundial de Alimentos (PMA)

El estado de la alimentación escolar a nivel mundial 2013. Roma: PMA. Recuperado de: <http://documents.wfp.org/stellent/ groups/public/documents/communications/ wfp262185.pdf >.

\section{Project Management Institute (PMI)}

2009 Guía de los fundamentos para la dirección de proyectos. Cuarta edición. Newtown Square.

\section{Project Management Institute (PMI)}

2013 Project Management Body of Knowledge. Quinta edición. Newtown Square.

\section{Project Management Institute (PMI)}

2018 Learn About PMI. Recuperado de: $<$ https://www.pmi.org/about/learn-aboutpmi>.

\section{Supply Chain Council (SCC)}

2005 Supply-Chain Operations Reference-Model.

Recuperado de: <http://people.ischool. berkeley.edu/ glushko/IS243Readings/

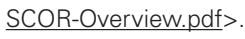

\section{Thompson, $\mathbf{R}$.}

s.f. Stakeholder analysis: Winning support for your projects. MindTools. Recuperado de: $<$ https://www.mindtools.com/pages/ article/newPPM 07.htm>.

\section{Tremolada, L. \& V. Valencia}

2014 Programa de Alimentación Escolar en Ventanilla: una gestión eficiente bajo la metodología SCOR. Proyecto profesional presentado para obtener el título profesional de licenciado en Gestión, con mención en Gestión Pública. Pontificia Universidad Católica del Perú. Lima, Perú.

\section{Ugarte, M.}

2011

Apuntes del curso regular de Gestión

Pública. Facultad de Gestión y Alta

Dirección de la Pontificia Universidad

Católica del Perú (PUCP). Lima: Pontificia

Universidad Católica del Perú.

\section{Weimer, D. \& A. Vining}

2011

Fecha de recepción: 01/07/2017

Fecha de aceptación: 17/01/2018 